\title{
A Novel Holistic Design Optimisation Algorithm for the Ironless Inductive Position Sensor
}

\author{
Adrian Grima ${ }^{1,2}{ }^{*}$, Mario Di Castro ${ }^{1}$, Alessandro Masi ${ }^{1}$, Nicholas Sammut ${ }^{2}$ \\ 1EN-SMM-MRO Group, European Organization for Nuclear Research (CERN), Geneva, Switzerland. \\ 2Department of Micro and Nanoelectronics, Faculty of ICT, University of Malta, Msida. \\ * Corresponding author. Tel.: +41 2276 78521; email: adrian.grima@cern.ch \\ Manuscript submitted May 14, 2018; accepted June 8, 2018. \\ doi: 10.17706/ijcee.2018.10.2.116-126
}

\begin{abstract}
The Ironless Inductive Position Sensor (I2PS) is a state-of-the-art high precision linear position sensor, which is designed to be radiation hard and immune to magnetic fields. This sensor is built for the Large Hadron Collider collimation system at the European Organization for Nuclear Research. It is continuously monitored to assess the precision, accuracy and drifts during the machine's operation. The ironless inductive position sensor was previously designed and optimised manually on a programmed electromagnetic model and simulated using a finite element model simulator. This sensor has the potential to be used extensively in industry, especially in areas with high radiation and high electro-magnetic interference. To industrialise it, an automated design procedure is required that offers the possibility to a user with minimal knowledge to design and optimise the sensor. This paper identifies the optimisation parameters and constants required in the manual design. It hence presents an automated design procedure which uses a multi-objective optimisation algorithm to automatically produce ironless inductive position sensors tailor-made to the user's specifications.
\end{abstract}

Key words: The ironless inductive position sensor (I2PS), genetic algorithm, multi-objective optimisation algorithm, optimisation algorithms.

\section{Introduction}

The Large Hadron Collider (LHC) [1]-[3] at The European Organisation for Nuclear Research (CERN) [4] is a safety-critical machine that demands unprecedented collimation specifications for its particle beams. Collimation sensors optimisation is required to: improve performance; reduce manufacturing; reduce sensor dimensions and reduce the cost. Hence, a compromise between cost, efficiency, size and performance needs to be found. Typically, in safety critical applications where high performance is required, the cost is given little importance. On the other hand, size is usually one of the hardest constraints to satisfy since in some applications the volume available is smaller than that required.

The Ironless Inductive Position Sensor (I2PS) [5], [6] is a novel sensor designed and optimised to be used as a valid alternative to the Linear Variable Differential Transformer (LVDT) in harsh environments (such as nuclear plants, plasma control or particle accelerators). While the approach to date is to have a design, which is as general as possible [7], all the dimensions and specifications used are chosen to have a perfect fit to substitute an LVDT in the LHC collimators.

The sensor is characterised in terms of high frequency [8] and thermal phenomena [9]. A procedure is also presented that explains how the sensor is optimised without the use of an automatic optimisation 
procedure [7]. The procedure involves a rigorous, manual, trial-and-error based method to obtain a set of design specifications for manufacturing. This approach has a number of disadvantages including: the need to have a deep understanding of the complex models and how to treat them which can only be done by a highly skilled user who knows the sensor`s design very well; the need for MathWorks Matlab software; high computational requirements and a long design time.

The general goal of this work is to industrialise the I2PS design tools such that they are used to their full potential by industry especially in environments, which have high radiation and electromagnetic interference. This paper presents the first step to fulfil this aim. It presents a novel approach with which to design an optimised ironless inductive position sensor with minimal resources. Furthermore, it highlights how the new approach makes use of an existing optimisation technique that uses a generalised programming language independent of proprietary software like Matlab. Contrary to the procedure currently used in [7] the optimisation design procedure presented in this paper is user-friendly and autonomous requiring minimal direct intervention by the user.

\section{The Ironless Inductive Position Sensor}

\subsection{The Structure of the I2PS}

Table 1. Physical Properties of the Sensor

\begin{tabular}{ll}
\hline \hline Parameter & Value \\
\hline Sensor Length & $235[\mathrm{~mm}]$ \\
Diameter & $13.4506[\mathrm{~mm}]$ \\
Sense- supply coil bobbin diameter & $19.2[\mathrm{~mm}]$ \\
Moving coil Bobbin diameter & $4[\mathrm{~mm}]$ \\
Sense - supply coils length & $80[\mathrm{~mm}]$ \\
Moving coil length & $90[\mathrm{~mm}]$ \\
Number of turns of the moving coil & 3150 \\
Number of turns of the supply coil & 4500 \\
Number of turns of the sense coil & 1388 \\
Moving coil wire diameter & $0.4[\mathrm{~mm}]$ \\
Sense coil wire diameter & $0.2[\mathrm{~mm}]$ \\
Supply coil wire diameter & $0.05[\mathrm{~mm}]$ \\
\hline \hline
\end{tabular}

The ironless inductive position sensor, like other linear position sensors, consists of two parts: the body of the sensor and the movable link. For the I2PS, the body is made up of four hermetically sealed air-cored cylindrical coils, which are protected from mechanical stress with a solid shield made of steel 316LN. The material for the shield is selected such that it is non-magnetic and exhibits high resistivity, in order to minimise eddy currents.

The four coils found in the body are wound on the same bobbin. This bobbin is hollow such that the movable link can go inside it. As shown in Fig. 1, the first two coils called the supply coils are wound next to each other and on top of the sense coils i.e. coils 3 and 4. Furthermore, coils 1 and 2 are wound in opposite directions. The supply coils are both connected in series and supplied with a sinusoidal current or they are both connected in parallel and supplied by a sinusoidal voltage. The sense coils are connected to the acquisition directly.

The movable link on the other hand is connected to the fifth coil which, as can be noted in Fig. 1. And Fig. 2 , is the innermost coil. The fifth coil is called the moving coil, which is the only coil wound on its own bobbin and not shielded. The material of this bobbin is non-metallic and is usually made out of plastic. A 
steel 316LN rod is used to link the moving coil to the collimator jaws. Typically, the diameter of this bobbin is kept as small as possible. Table 1 gives the physical parameters of the sensor. As shown, the sensor is made up of very fine wires which result in very thin coils.

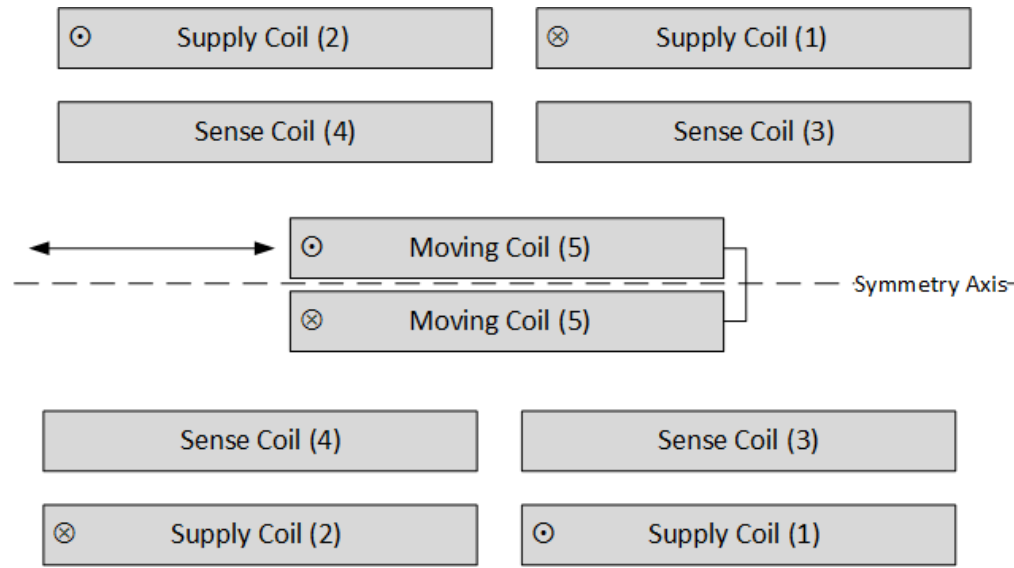

Fig. 1. Longitudinal cross-section of the ironless inductive position sensor structure.

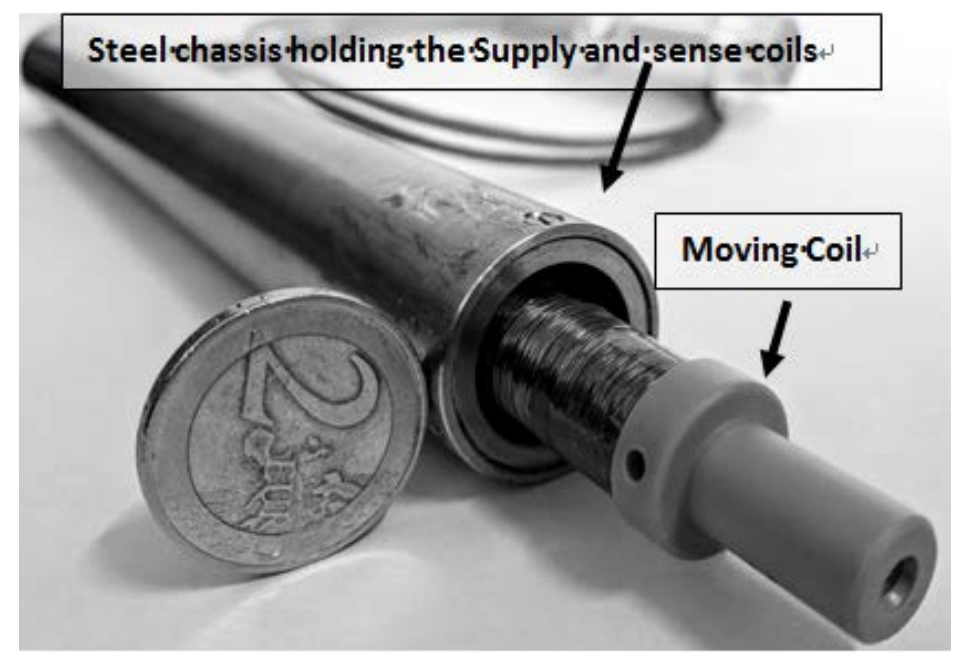

Fig. 2. The ironless inductive position sensor.

\subsection{The Working Principle of the I2PS}

The I2PS sensing structure is based on inductive coupling. The moving coil is short-circuited such that there is an induced current in this coil as a result of a changing magnetic field generated by the supply coils. When the moving coil is at the centre of the sensor, the net induced current is zero. As the mutual inductances between the moving coil and the two supply coils are equal, the sum of the induced currents becomes zero. Hence, the voltages read from the two sense coils are equal. This is the equilibrium point.

This symmetry is broken when the moving coil moves away from the centre. Since the moving coil is physically nearer to one of the two supply coils, the total flux linked to the winding is not zero anymore. Consequently, the induced current in the moving coil is also not zero. According to Faraday's law, this generates an additional magnetic field, counteracting the one inducing the current. Consequently, the magnetic coupling with the sense winding is disrupted, leading to two different amplitudes for the sense voltages. To be precise; the induced current leads to a counter-acting magnetic field, which adds up with the supply field. As a result, the total flux concatenated with the sense windings is affected by the presence of the moving coil's counter-acting field. The core position is hence obtained by a differential reading of these 
voltages. Therefore, in this way, a variable magnetic flux according to the position is created and, at the same time, a linear relationship between magnetic field and magnetic flux density (i.e. constant differential permeability) is maintained, leading to an intrinsic immunity to external fields.

\subsection{The Mathematical Model}

An electromagnetic model is defined in [10]. To summarize, the overall mutual inductance between two coils at a distance $c_{o}$ can be calculated by:

$$
M_{T}\left(c_{o}\right)=\mu_{o} \sqrt{R_{p} R_{S}} \sum_{i=1}^{N_{l p}} \sum_{j=1}^{N_{l s}} \sum_{n=1}^{N_{p}} \sum_{l=1}^{N_{s}} \phi_{i, j, n, l}\left(k_{i, j, n, l}\right)
$$

where :

$$
k_{i, j, n, l}^{2}=\frac{4 \alpha_{i, j}}{\left(1+\alpha_{i, j}\right)^{2}+\beta_{i, n, l}^{2}}
$$

The geometrical values $R_{s}$ and $R_{p}$ are the coil radii are a function of the layer and/or of the distance between the single circular turns, as follows.

$$
\alpha_{i, j}=\frac{R_{s, j}}{R_{p, i}}, \quad \beta_{i, n, l}=\frac{C_{n, l}}{R_{p, i}}
$$

with

$$
\begin{gathered}
R_{p, i}=R_{p}+(i-1) d_{p}, \quad R_{s, j}=R_{s}+(j-1) d_{s} \\
c_{n, l}=c_{0}+\left(z_{n}-z_{l}\right) \\
z_{n}=-a_{p}+(n-1) d_{p}, \quad z_{l}=-a_{s}+(l-1) d_{s}
\end{gathered}
$$

where:

$a_{p}$ and $a_{s}$ represent the semi-length of the first and second coil respectively

$d_{s}$ and $d_{p}$ are the corresponding wire diameters

$z_{n}$ and $z_{l}$ are the z-coordinates corresponding to the $n^{\text {th }}$ and $i^{\text {th }}$ turn of the first and second winding respectively.

$N_{l p}, N_{l s}$ are the number of layers of the first and second winding and $N_{p}, N_{s}$ are the number of turns per layer of the first and second winding respectively.

$$
\phi(k)=\left(\frac{2}{k}-k\right) K(k)-\frac{2}{k} E(k)
$$

where $K$ and $E$ are the complete elliptic integrals of first and second kind respectively [11].

Since, the moving coil is short-circuited, the induced current can be obtained by considering all the magnetic fluxes (obtained by using ( 1 )) coupled with the coil and apply the voltage balance on its mesh. Hence, the induced current $\mathrm{I}_{5}$ in the moving coil is:

$$
I_{5}=-\frac{j \omega}{Z_{5}}\left(M_{51} I_{1}+M_{52} I_{2}\right)
$$

where

$Z_{5}=R_{5}+j \omega L_{5}$ is the winding impedance

$M_{i j}$ is the mutual inductance between windings $i$ and $j$.

$I_{i}$ is the current in coil $i$, since the current in coil 1 and 2 is the same then $\mathrm{I}=-\mathrm{I}_{1}=\mathrm{I}_{2}$

The sense coils' voltages for the I2PS are:

$$
\begin{aligned}
& V_{3}=j \omega\left(M_{31}-M_{32}\right) I+j \omega M_{35} I_{5} \\
& V_{4}=j \omega\left(M_{41}-M_{42}\right) I+j \omega M_{45} I_{5}
\end{aligned}
$$


where:

$V_{3}$ is the voltage output from one of the sense coils of the I2PS,

$M_{i j}$ are the mutual inductances between $i^{\text {th }}$ and $j^{\text {th }}$ coils,

$I$ is the input current (since the I2PS is using a current supply) and

$I_{5}$ is the current induced in the moving coil.

The basis of this model is the calculation of the inductance between two circular conductors using elliptic integrals. This model needs the conductors to be coaxial but not concentric. Another function then calculates the mutual inductance between two circular solenoids. These two functions are then used iteratively with different inputs to calculate the mutual inductance between the different layers, turns of the coils and the position of the moving coil. The same functions are also used when the self-inductance of a thick wall solenoid is calculated. Finally, the mutual inductances of the model are used to calculate the voltages and currents in the coils.

The I2PS model is very complex hence, the sensor's models were originally written in Matlab, which offers a wide variety of functions and is easy to use. Due to the repetitive and looped use of the elliptic integrals, this model also uses a high amount of computational resources making the algorithm time consuming.

\section{The Optimisation Problem}

The I2PS prototype available was designed manually and manufactured with the aim to substitute a number of LVDTs found in the LHC collimators. The aim is to have a design tool that uses the theoretical models to design and optimize a new sensor for any application defined by the user. This tool must be able to accept the parameters defined by the user, suggest where possible calculated values and make the necessary checks such that the sensor design is viable. At the end depending on the inputs, the tool must find the optimal solution.

The number of design parameters listed in the previous sub-section makes the optimisation process non-trivial. As can be noted from Table 2 and Table 3, some parameters are dependent on several others, so much so that the influence on the objective function cannot be simply estimated by considering them individually and sequentially. Furthermore, implementing a sequential trial and error-based system requires multiple iterations of the same stage. Moreover, the computation of the model involves the evaluation of elliptic integrals of first and second kind hence involving the numerical computation of these integrals. It also requires the computation of non-linear operations and has parameters that have to be only integer numbers such as the number of layers and the number of turns.

\subsection{Parameters, Constraints and Dependencies}

Since the design tool is built for a generic application without any guidelines to follow, a holistic approach is required when defining what is constant and what needs to be varied in order to produce an optimised sensor.

Table 2. List of I2PS Constants

\begin{tabular}{ll}
\hline \hline Constant & Dependency \\
\hline Geometrical size & The area of application \\
Bobbin \& insulation & The manufacturer \\
Max supply V/I & The Supply Board /Source \\
Max Sense Voltage & The acquisition board selected/available \\
Max № of layers & The manufacturer \\
Survey time & The acquisition system \\
\hline \hline
\end{tabular}

Table 2 identifies the sensor's constants. The geometrical constraints set by the user, especially the 
sensor's diameter, will influence all coils' diameters. The length of the sensor will affect the coils' length and connectors, and hence the maximum range. The bobbin and insulation minimum thickness that can be used also affects the coils' diameters and hence the max number of turns possible.

The survey time affects the operating frequency and readout parameters such as sampling frequency, number of samples etc. Moreover, the coils' voltage must respect the data acquisition channels' range and guarantee a good resolution of the conditioning electronics. This will have impact on the choice of the frequency and excitation signal amplitude.

Table 3. Variables to Be Optimised and Their Respective Dependencies

\begin{tabular}{|c|c|}
\hline Variables & Dependency \\
\hline $\begin{array}{l}\text { The number of layers of the supply coil } \\
\text { (NLP) }\end{array}$ & The diameter of the sensor and sense coil diameter \\
\hline $\begin{array}{l}\text { The number of layers of the sense coil } \\
\text { (NLS) }\end{array}$ & $\begin{array}{l}\text { The diameter of the sensor, the bobbin diameter and the max air gap allowed } \\
\text { between MC and bobbin }\end{array}$ \\
\hline $\begin{array}{l}\text { The number of layers of the moving } \\
\text { coil(NLC) }\end{array}$ & The diameter of the sensor and moving coil bobbin radius \\
\hline $\begin{array}{l}\text { The semi-length of the supply (as) and } \\
\text { sense coil (ap) }\end{array}$ & The length of sensor \\
\hline The semi-length of the moving coil (ac) & $\begin{array}{l}\text { The length of sense and supply coils. The non-linearity error and the sensor's } \\
\text { sensitivity }\end{array}$ \\
\hline The supply coil wire diameter & The sensor's diameter and ampacity \\
\hline The sense coil wire diameter & The sensor's diameter \\
\hline The moving coil wire diameter & $\begin{array}{l}\text { The sensor's diameter \& resistivity (must be as low as possible due to high } \\
\text { frequency effects) }\end{array}$ \\
\hline
\end{tabular}

The NLP, NLS, NLC and ac will influence the output voltage produced, the sensitivity of the sensor and the Non-Linearity Error (NLE) [12]. The NLE is computed as

$$
N L E=100 \frac{\max \left(p^{\prime}-p *\right)}{C P R}
$$

where $p^{*}$ is the core reference position, $\mathrm{p}^{\prime}$ is the position calculated through linear interpolation of the curve and CPR is the core position range. In [13], it was found out that the optimum choice for the coils length would be of $\geq 2 P_{\max }$, where $P_{\max }$ is the maximum range required. In addition it was also shown that the best ratiometric swing is given when the length of all coils is equal ( $a s=a c=a p)$. Hence, the ratiometric swing, the extreme position effects and geometrical constraints impact the coils' lengths.

The diameter of the supply cable is calculated depending on the supply current / voltage and the current rating of the wire diameter selected. On the other hand, the model assumes there is no current in the sense coils due to the high impedance readout system. This implies that no current density calculation is needed for these coils. Furthermore, the sense coil needs to have a high amount of turns since the sensitivity is dependent on the number of turns of the sense coil and that of the moving coil. Hence, the diameter of the sense cable is selected to be the thinnest possible. The diameter of the moving coil is optimised depending on the space required and output voltage produced and the sensitivity obtained. While a high number of turns is recommended for good sensitivity the resistance of the moving coil must be kept low enough for current to be induced. A high resistance value impacts both the sensitivity and the output voltage.

\subsection{The Procedure}

Designing an I2PS requires a multi-objective [14], [15] optimisation algorithm. Furthermore, the user is required to input the constants depending on the dependencies and is required to select the weighting and the number of recombinations. It can also be understood that the objectives targeted at this point can be increased to the extent that new objects, such as costs, can also be included. Various multi-objective 
methods have been identified and studied. Evolutionary algorithms[16], however, are a very popular approach to obtain multiple solutions in a multi-modal optimization task [17]. The genetic algorithm (GA) method [18]-[22] is selected due to the ease of implementation with respect to other methods, such as the normal boundary intersection[23] or the multi-objective particle swarm optimisation[24], [25]. In this case, the chromosomes used are made of real numbers and are of a fixed-length. Since the variable of the parameter space is continuous, a real-coded GA is coded rather than a binary one. This algorithm, which is programmed in $\mathrm{C}++$, works in the following manner:

1) A population of 10 genotypes is randomly generated each with four chromosomes. The random generation is based on a random device, which produces non-deterministic random numbers combined with a uniform integer or real distribution. This distribution produces random integers in a range set where each possible value has an equal likelihood of being produced.

2) Each population is tested for space requirements and if it does not fit, a new population is generated. This allows the algorithm to discard bad spatial combinations immediately and add diversity to the generation.

3) The I2PS model is used to calculate the output values and a fitness is assigned to each gene. The fitness is the difference between the target value and the available value.

4) The fitness values are normalised. Values out of bounds are given negative values. The normalisation is set such that the values close to the target are closer to one.

5) Weighted sums based on the user's input in the graphical user interface (GUI) is used to calculate the final fitness function combining all the other fitnesses.

6) The genes are then sorted in descending order from the best to the worst fitness function.

7) The packing method is used to check for any duplicates.

8) The best fitness is kept while all the others, apart from the ones generated with packing method, are newly generated from crossover and mutation. Single point crossover is implemented and random offspring generation is used in case the same gene is selected for both parents. Mutation is done in a fair coin toss method at a randomly generated chromosome.

9) The procedure repeats until the set number of recombinations is reached or the optimal design is found.

To ensure diversity and hence prevent premature convergence to local optima [26], the algorithm makes use of the social disasters technique. Kureichick et al. introduced this technique in [27]. This technique's sole purpose is to return the population to an acceptable degree of genetic diversity, by replacing a number of selected individuals, by others generated at random. The packing and the judgment day methods are the two different methods considered and used in the I2PS optimisation algorithm. In the packing method, all the individuals that have the same fitness value are destroyed except one. This means that only one remains unchanged while all the others are fully randomized. In the judgment day method, only the individual with the best fitness value remains unchanged while all the others are fully randomized.

\section{Results}

The application outputs the best two combinations found as well as a list of the top combinations considered along the process. The tool also outputs all the dimensions of the sensor for prototyping. It allows the user to calculate the electrical parameters and generates a spice command package to simulate the sensor in spice.

As a test, the tool is run with 50 recombinations and with the judgment day protocol activated. It is set to maximise the output voltage (in this case set to $10 \mathrm{~V}$ ) and position range (the moving coil length - $a c$ ). Furthermore, the moving coil length is set to stay within $\pm 10 \mathrm{~mm}$ of the nominal value (as found in [28]), 
which states that all the coils should have the same length. The same parameters as the manual optimisation method used before are set. This same test was repeated multiple times to also test for convergence to the same design parameters.

Fig. 3. shows the result of a run. The higher the fitness function, the closer the design of the sensor is to reaching the required optimum. A low fitness function means the design combination results in a design that falls outside of the design requirements.

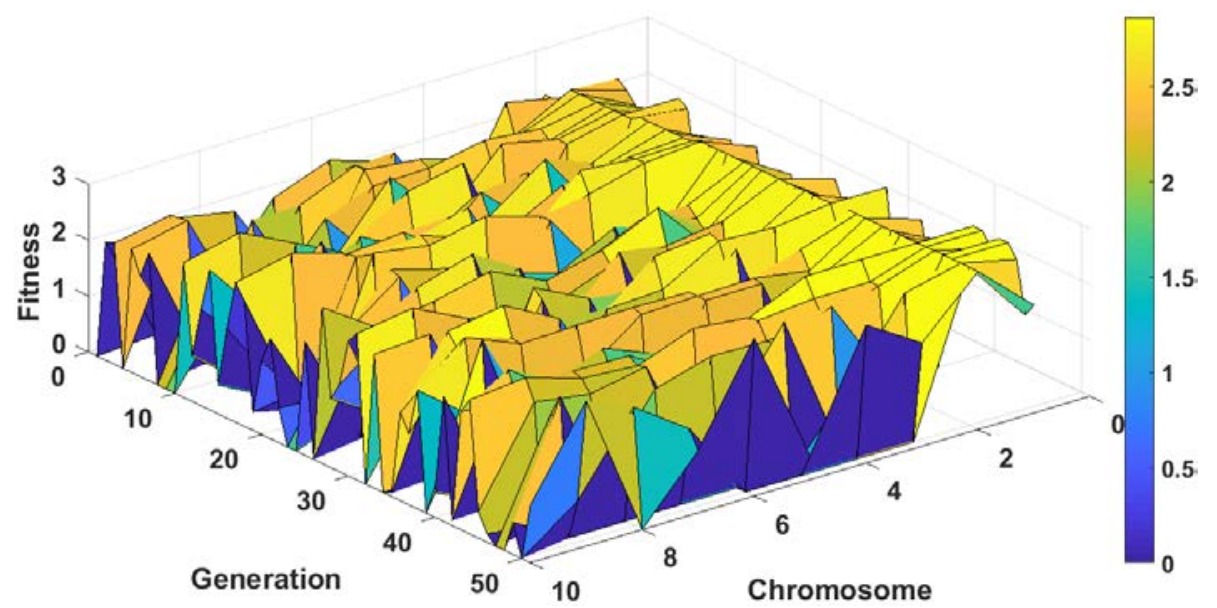

Fig. 3. 3D plot representing the fitness of each chromosome for each generation.

From the figure, it is also noted that chromosomes 6 to 10 are continuously changing values; this is because they are usually the newly generated combinations. The first (1-5) chromosomes are the ones that have a fitness function, which always increases i.e. the design parameters, are improved. The second chromosome is the fittest one of the group. Furthermore, generations, which seem to be converging towards the fittest chromosome, can also be seen. This happens since every generation starts to crossover with similar combinations hence, becoming similar and converging towards a possible local maximum. This is avoided with the judgment day protocol where only the fittest is kept.

As can be noted from Table 4, the best fitness assigned in this case had a very high value of output voltage, and a relatively good Non-Linearity Error (NLE) and sensitivity while the moving coil length used is close to the nominal. A clearer example of how the algorithm is considering everything is probably seen in the third and fourth values. The third has lower output voltage, and higher NLE, and the sensitivity is degraded with respect to that of the fourth place yet the ac (the moving coil length) is closer to the optimum and hence it is assigned a higher fitness.

Table 4. Example of a Combination, with Input / Output Values and Fitness

\begin{tabular}{lllllllll}
\hline \hline № & Nlp & Nls & Nlc & ac [mm] & V [V] & NLE [\%] & Sensitivity & Fitness \\
\hline 1 & 3 & 4 & 12 & 87.6 & 9.8 & 0.5 & -217 & 2.4 \\
2 & 3 & 3 & 14 & 77.0 & 8.5 & 0.7 & -201 & 2.3 \\
3 & 3 & 5 & 5 & 88.3 & 6.3 & 0.4 & -304 & 2.0 \\
4 & 4 & 2 & 13 & 95.9 & 7.0 & 0.3 & -215 & 2.0 \\
5 & 3 & 5 & 4 & 91.6 & 5.6 & 0.3 & -329 & 1.8 \\
6 & 3 & 4 & 4 & 91.7 & 4.4 & 0.3 & -326 & 1.7 \\
7 & 3 & 1 & 11 & 75.5 & 2.2 & 0.8 & -218 & 1.5 \\
8 & 5 & 3 & 10 & 84.9 & 10.2 & 0.6 & -230 & 1.2 \\
9 & 4 & 5 & 7 & 98.4 & 10.4 & 0.3 & -279 & 0.6 \\
10 & 5 & 5 & 10 & 98.5 & 17.4 & 0.3 & -244 & -6.3 \\
& & & & & & & & \\
$\mathrm{M}$ & 4 & 3 & 14 & 90 & 9.8 & 0.3 & -240 & \\
\hline \hline
\end{tabular}


It can be noted how output voltage values higher than the maximum set are given low importance. A tolerance is set where if the output voltages are greater than $\pm 0.5 \mathrm{~V}$ of the specified maximum, their respective fitness value is set to negative. This is immediately eliminated in the next generation.

Comparing the best result (№: 1), in Table 4, with manually optimised sensor (№: M), the number of layers vary but are close. The number of layers and hence the number of turns for the supply and sense coils are swapped. In reality, this can be more advantageous from a manufacturing point of view since it is easier to add a uniform layer with a very thin wire than a thicker wire. Similarly, for the number of turns of the moving coil, it is more beneficial to limit the number of layers. The maximum output voltage is similar. This is the value that the design tool gives priority to. On the other hand, the non-linearity error and the sensitivity are degraded. This is due to the parameters of the moving coil. Since the moving coil is selected to be shorter and with less number of turns the linearity close to the end of the sensor decreases as does the sensitivity.

\section{Conclusion}

The ironless inductive position sensor prototype is a state-of-the-art linear position sensor that has proven to be immune to electromagnetic interference during operation in CERN's LHC collimators. The design is robust, with a long lifetime and with very high sensitivity to position change. The current design process involves a long, iterative and manual procedure where the user needs to take all the decisions.

This paper presents a novel weighted multi-objective genetic algorithm that optimises the sensor's parameters with minimal user intervention. The advantage of this new procedure is that through the multi-objective optimisation algorithm, an automated holistic approach is taken when designing the new sensor. This allows all parameters to be optimised at the same time. Furthermore, due to the migration to a generalised programming language the computational resources required have reduced drastically. The results show that with a small number of re-combinations, i.e. in a short execution time of about 20 minutes, a similar design to the one optimised manually is obtained. Since, a very rigorous and lengthy manual design was performed in the past with the manual method; an automated result close to the former is accepted. The value added is a comparable design that can be generated very quickly by a user with minimal training.

\section{References}

[1] Brüning, O., Collier, P., Lebrun, P., Myers, S., Ostojic, R., Poole, J., \& Proudlock, P. (2004). Large hadron collider design report. Geneva: CERN: European Organization for Nuclear Research, 548.

[2] Evans, L., \& Bryant, P. (2008). LHC machine. Journal of Instrumentation, 3(8), S08001.

[3] Assmann, R., et al. (2005). LHC collimation: Design and results from prototyping and beam tests. Proceedings of the Particle Accelerator Conference.

[4] Lefevre, C. (2008). The CERN accelerator complex. CERN PhotoLab: Physics Diagrams and Charts.

[5] Danisi, A. (2013). Ironless inductive position sensor for harsh magnetic environments. École Polytechnique Fédérale de Lausanne.

[6] Grima, A., et al. (2017). Influence of external conductive objects on the performance of an ironless inductive position sensor. IEEE Sensors Journal, 17(14), 4500-4507.

[7] Danisi, A., et al. (2013). Design optimization of an ironless inductive position sensor for the LHC collimators. Journal of Instrumentation, 8(9), P09005.

[8] Danisi, A., et al. (2013). Modelling of high-frequency electromagnetic effects on an ironless inductive position sensor. IEEE Sensors Journal, 13(12), 4663-4670.

[9] Danisi, A., et al. (2014). Modelling and compensation of thermal effects on an ironless inductive 
position sensor. IEEE Trans. Ind. Appl., 50(1), 375-382.

[10] Danisi, A., et al. (2013). Electromagnetic analysis and validation of an ironless inductive position sensor. IEEE Transactions on Instrumentation and Measurement, 62(5), 1267-1275.

[11] Abramowitz, M., \& Stegun, I. A. Handbook of mathematical functions: With formulas, graphs, and mathematical tables. Courier Corporation, 196455.

[12] Nyce, D. S. (2004). Magnetostrictive Linear Position Sensors: Theory and Application. Hoboken (NJ): John Wiley and Sons.

[13] Danisi, A. (2013). Ironless inductive position sensor for harsh magnetic environments. EPFL Scientific Publications.

[14] Deb, K. (2011). Multi-Objective Optimization Using Evolutionary Algorithms. Hoboken (NJ): John Wiley \& Sons.

[15] Weise, T. (2009). Global optimization algorithms-theory and application. Proceedings of the XII Global Optimization Workshop Mago, vol. 7445, no. 2 (pp. 242-243).

[16] Zitzler, E., \& Thiele, L. (1998). An evolutionary algorithm for multi-objective optimization: The strength pareto approach. TIK-Report, 43.

[17] Contributors, W. (2018). Mathematical optimization - Wikipedia, the free encyclopedia.

[18] McCall, J. (2005). Genetic algorithms for modelling and optimization. J. Comput. Appl. Math., 184(1), 205-222.

[19] Contributors, W. (2018). Genetic algorithm — Wikipedia, The Free Encyclopedia.

[20] Circiu, M. S., \& Leon, F. (2010). Comparative study of multi-objective genetic algorithms. Buletinul Institutului Politehnic Din Iaşi.

[21] Mitchell, M. (1998). An Introduction to Genetic Algorithms. DBLP, 188. Cambridge: MIT Press.

[22] Gen, M., \& Cheng, R. (2007). Genetic algorithms and engineering optimization. Hoboken (NJ): John Wiley \& Sons.

[23] Das, I., \& Dennis, J. E. (1998). Normal-boundary intersection: A new method for generating the Pareto surface in nonlinear multi criteria optimization problems. SIAM Journal on Optimization, 8(3), 631-657.

[24] Kennedy, J. (2011). Particle swarm optimization. Encyclopedia of Machine Learning Springer.

[25] Chen, G., \& Yu, J. (2005). Particle swarm optimization algorithm. Information and Control-Shenyang, 34(3), 318.

[26] Rocha, M., \& Neves, J. (1999). Preventing premature convergence to local optima in genetic algorithms via random offspring generation. Proceedings of the International Conference on Industrial, Engineering and Other Applications of Applied Intelligent Systems.

[27] Kureichick, V., et al. (1996). Some new features in genetic solution of the travelling salesman problem. Adaptive Computing in Engineering Design and Control.

[28] Danisi, A., et al. (2013). Modelling of moving coil capacitance in an ironless inductive position sensor.

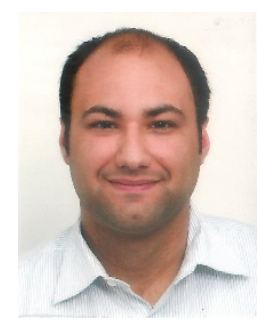

Adrian Grima was born in Rabat, Gozo, Malta, in 1992. He received the B.Eng. (hons) degree in electrical and electronics engineering, the M.Sc. degree in microelectronics and nano electronics from the University of Malta, Msida, Malta, in 2015. He is currently working toward the Ph.D degree at the University of Malta. In 2012 and 2013, he carried out research activities with regard to Infra-red reflectography (IRR) for the analysis of historical paintings. Since 2014, he has been affiliated with the Department of Microelectronics and Nanoelectronics and the University of Malta and with the Sources, Targets and Interactions Group in the Engineering Department, European Organization for Nuclear Research (CERN), carrying on research on electromagnetic modelling and characterization of sensors in the 
presence of electro-magnetic fields. His main research interests are in the field of new sensor design, sensor characterisation and instrumentation.

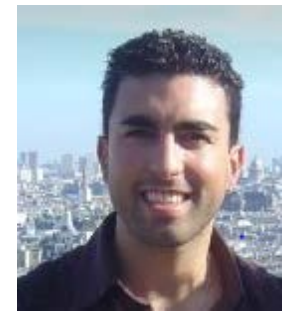

Mario Di Castro received the M.Sc. degree in electronic engineering from the University of Naples "Federico II", Italy. From October 2007 until September 2011 he has worked at DESY in charge of robotic controls and industrial automation. Since October 2011 he works for CERN and since February 2018 he leads the mechatronics, robotics and operation section in the Engineering Department. Important section activities are the design, installation, maintenance and controls of complex mechatronics and robotics systems, as well as the operation of the beam intercepting devices of the CERN accelerator complex.

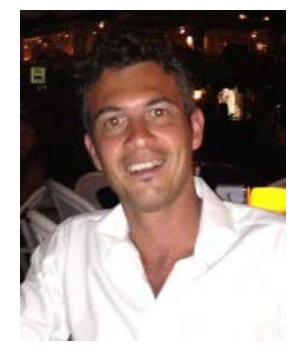

Alessandro Masi received the M.Sc. degree in electronic engineering and the Ph.D. degree in computer science from the University of Naples "Federico II", Italy. Since April 2005, he is a CERN Staff Member and since January 2008, he has leaded the equipment controls and electronics section in the Source, Targets, and Interactions Group. Important section activities are design, installation, and maintenance of control systems.

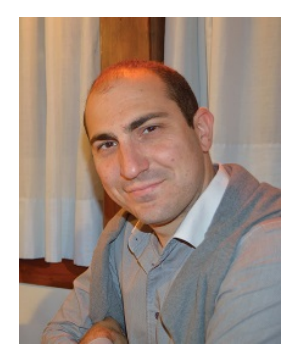

Nicholas Sammut was awarded a B.Eng. (hons) degree in electrical engineering, an M. Ent. degree in entrepreneurship and the Ph.D. in electrical engineering from the University of Malta. His Ph.D. research on the field description of the large hadron collider was carried out with the Magnetic Measurements and Tests Group, European Organisation for Nuclear Research (CERN), Geneva, Switzerland. He is an academic in the Department of Microelectronics and Nanoelectronics at the University of Malta since 2007. He also serves as deputy dean of the Faculty of Information and Communications Technology of the same university. He served as a board member on several Maltese, European and international organisations and committees. His main research interests are in the field of accelerator technology, sensors, instrumentation and measurement. 